\section{ASPECTOS DE LAS LEGUMBRES NUTRICIONALES Y BENEFICIOSOS PARA LA SALUD HUMANA}

\author{
Cristina Delgado-Andrade \\ Consejo Superior de Investigaciones Científicas \\ Raquel Olías \\ Consejo Superior de Investigaciones Científicas \\ Jose Carlos Jiménez-López \\ Consejo Superior de Investigaciones Científicas \\ Alfonso Clemente \\ Consejo Superior de Investigaciones Científicas \\ alfonso.clemente@eez.csic.es
}

Cómo citar este artículo/Citation: Delgado-Andrade, C.; Olías, R.; Jiménez-López, J. y Clemente, A. (2016). Aspectos de las legumbres nutricionales y beneficiosos para la salud humana. Arbor, 192 (779): a313. doi: http://dx.doi.org/10.3989/ arbor.2016.779n3003

Recibido: 08 marzo 2016. Aceptado: 06 mayo 2016.

RESUMEN: Las leguminosas constituyen una de las familias botánicas más importantes desde el punto de vista nutricional, siendo un componente importante de la dieta mediterránea y esencial en la de numerosos países en desarrollo. Legumbres tales como garbanzo, lenteja, altramuz, guisante y habas son reconocidas como fuentes de proteínas, almidón, fibra, vitaminas y minerales. En este trabajo, quedan incluidas las evidencias científicas relativas al papel que juegan las legumbres en la nutrición humana así como las propiedades preventivas que ejercen algunos de sus componentes bioactivos en enfermedades tales como la diabetes tipo-2, hipercolesterolemia, hipertensión, obesidad, enfermedades inflamatorias y cáncer. Por último, se analiza los datos de consumo de legumbres en los hogares españoles, los cuales demuestran un descenso superior al $60 \%$ en las últimas décadas. Esto debe ser corregido con políticas de promoción de dieta saludable así como por medidas de adaptación al nuevo mercado con la aparición de nuevos productos que vayan orientados a las preferencias actuales del consumidor.

PALABRAS CLAVE: legumbres; nutrición humana; compuestos bioactivos; salud; prevención; inflamación; cáncer; diabetes tipo-2; hipercolesterolemia; consumidor.

\section{NUTRITIONAL AND BENEFICIAL EFFECTS OF GRAIN LEGUMES ON HUMAN HEALTH}

Copyright: (C) 2016 CSIC. Este es un artículo de acceso abierto distribuido bajo los términos de la licencia Creative Commons Attribution (CC BY) España 3.0.

ABSTRACT: The Leguminosae is one of the most important botanical families from a nutritional perspective, constituting one of the main ingredients in the Mediterranean diet and an essential food in developing countries. Grain legumes like chickpea, lentil, lupin, pea and beans are well known sources of proteins, starch, fiber, vitamins and minerals. In this paper, scientific evidence for the role of legumes in human nutrition, as well as the preventive properties of some of their bioactive components in diseases like type-2 diabetes, hypercholesterolemia, hypertension, obesity, inflammatory diseases and cancer, are considered. Finally, data of consumption in Spanish households was analyzed, where a $60 \%$ decrease has been observed over the last decades. This trend must be reversed by implementing policies to promote healthy diet and by adapting to a new market by means of developing novel products oriented to consumer preferences.

KEYWORDS: grain legumes; human nutrition; bioactive compounds; health; prevention; cardiovascular disease; inflammation; cancer; type-2 diabetes; hypercholesterolemia; consumer. 


\section{INTRODUCCIÓN}

Las leguminosas constituyen unas de las familias botánicas más relevantes desde el punto de vista nutricional, siendo un componente importante de la dieta mediterránea y esencial en la de numerosos países en desarrollo. Éstas se consumen como semillas secas, limpias y separadas de la vaina. Las leguminosas destinadas a consumo humano quedan divididas en tres grandes grupos: leguminosas grano, proteaginosas y oleaginosas. Dentro del grupo de las leguminosas grano, destacan las judías secas (Phaseolus vulgaris), garbanzos (Cicer arietinum) y lentejas (Lens culinaris). Los guisantes (Pisum sativum), las habas (Vicia faba) y el altramuz dulce -donde se incluyen Lupinus angustifolius, L. albus y L. luteus- quedan englobados en el grupo de las proteaginosas mientras que la soja (Glycine max) se encuentra enmarcada en el grupo de las oleaginosas. De manera general, podemos afirmar que las leguminosas son una fuente de carbohidratos y proteína de buena calidad, mostrando un contenido elevado de fibra. Las leguminosas contienen un bajo contenido en lípidos (siendo la excepción la soja y el cacahuete), niveles fisiológicamente relevantes de ciertos minerales tales como calcio, magnesio, potasio, hierro y zinc, así como de vitaminas, especialmente vitaminas del complejo $\mathrm{B}$ y ácido fólico. La Tabla 1 muestra la composición nutricional de las leguminosas de mayor consumo humano, según recoge la base de datos del Departamento de Agricultura de Estados Unidos (USDA National Nutrient Database for Standard Reference Release 28, slighlty revised in May 2016).

Un número creciente de estudios postulan que el consumo de legumbres está asociado a propiedades beneficiosas en salud, atribuidas al contenido elevado en fibra -soluble e insoluble- y de almidón resistente a la degradación durante el proceso digestivo, así como a la presencia de algunas proteínas con propiedades bioactivas y compuestos polifenólicos. Además, las legumbres presentan compuestos con propiedades potencialmente beneficiosas para la salud, tales como taninos, saponinas, fitatos, lectinas o inhibidores de proteasas que también pueden ser considerados factores antinutritivos, ya que reducen la absorción de nutrientes, disponibilidad mineral y digestibilidad proteica. En este trabajo, quedan incluidas las evidencias científicas sobre el papel beneficioso que ejerce el consumo de legumbres en la prevención de enfermedades tales como la diabetes-tipo2, hipercolesterolemia, hipertensión, enfermedades inflamatorias y cáncer. Además, se incluyen los hábitos de consumo de legumbres en los hogares españoles así como las recomendaciones de ingesta establecidas por organizaciones de salud.

\section{COMPOSICIÓN QUÍMICA DE LAS LEGUMBRES}

\subsection{Proteínas}

Las legumbres se caracterizan por su elevado contenido proteico, muy superior al de los cereales. El contenido proteico presenta una gran variabilidad ( $20 \%$ en guisante y $38-40 \%$ en altramuz y soja), siendo dependiente de la especie, genotipo y factores medioambientales. Las globulinas, proteínas de reserva de los cotiledones que son sintetizadas durante el periodo de formación de la semilla para ser posteriormente utilizadas como fuente de energía, carbono y nitrógeno a lo largo de proceso de germinación y crecimiento de la plántula, son los principales constituyentes proteicos de las leguminosas (65-80\% del total proteico). Las albúminas, aunque cuantitativamente menos importantes que las globulinas (15-25 \% del total de proteínas) juegan un papel biológico destacado en las semillas de las legumbres ya que están constituidas fundamentalmente por enzimas y proteínas citoplasmáticas funcionales. En esta fracción proteica quedan englobadas proteínas tales como las lectinas, inhibidores de amilasa y de proteasas, entre otras, con potencial nutracéutico (Duranti, 2006).

Respecto a la composición de aminoácidos, ésta se caracteriza por niveles bajos de azufrados (metionina y cisteína) y elevados de lisina; esto hace que las proteínas de legumbres se complementen con proteínas de cereales. La calidad nutricional de las proteínas está determinada por los requerimientos de aminoácidos de nuestro organismo y su disponibilidad al ser digeridos (FAO, 2013). De manera general, las legumbres presentan una calidad proteica inferior a las de proteínas de origen animal, tales como las de la leche o el huevo. Esto puede ser debido a diversos factores, que incluyen su bajo nivel en aminoácidos azufrados, la resistencia a digestión de ciertas proteínas -relación estructura-digestibilidad- y la presencia de compuestos antinutritivos que dificultan la digestibilidad de las proteínas. Además de su calidad proteica per se, estas proteínas contienen péptidos bioactivos con propiedades beneficiosas para la salud, pudiendo formar parte de formulaciones infantiles y clínicas (Clemente, 2000).

\subsection{Carbohidratos}

El almidón, la fibra y los oligosacáridos constituyen los componentes principales de carbohidratos presentes en las legumbres. Éstas tienen un elevado contenido en carbohidratos, siendo el almidón el polisacárido mayoritario (35-45 \% del peso seco de las semillas), excepto en soja donde su contenido es bajo (Salunke y 
Kadam, 1989). El almidón está constituido fundamentalmente por amilosa y amilopectina; a diferencia del almidón de cereales, el de las legumbres se caracteriza por el predomino de la fracción de amilosa (30-40\%), responsable de su baja digestibilidad debido a que tras la cocción ésta actúa como almidón resistente (Guillon y Champ, 2002); de esta forma, escapa a la acción de las enzimas digestivas y alcanza el colon, donde es parcialmente fermentado por la microbiota intestinal y/o eliminado en las heces (Ros y Periago, 2005). La fermentación colónica del almidón resistente por parte de la microbiota intestinal produce como principales metabolitos ácidos grasos de cadena corta que muestran propiedades beneficiosas en salud (Fernández, Redonde-Blanco, Villar, Clemente y Lombó, 2015).

Las legumbres presentan un alto contenido en fibra dietética (14-33\%) (Vaz Patto et al., 2015), lo cual contribuye al bajo índice glicémico de las legumbres (Messina, 2014). Químicamente, la fibra está constituida por un conjunto de moléculas heterogéneas, tales como hemicelulosa, pectinas y lignina derivada de carbohidratos estructurales procedentes de la pared celular. La cubierta de las semillas contienen niveles elevados de celulosa mientras que los cotiledones presentan niveles elevados de pectinas, celulosa y glicanos (Guillon y Champ, 2002). Dependiendo de su solubilidad en agua y soluciones tamponadas, la fibra se clasifica en soluble e insoluble. Los niveles de fibra soluble de las legumbres son superiores a los encontrados en cereales y tubérculos y se encuentra constituida fundamentalmente por pectinas, gomas, mucílagos, algunas hemicelulosas y polisacáridos. Como ejemplo, se ha documentado que hasta un $25 \%$ de la fibra total de las judías secas es de carácter soluble, frente a los bajos porcentajes detectados en el trigo (Ros y Periago, 2005). La fibra soluble es resistente al proceso digestivo y escapa de la absorción intestinal, siendo parcial- o totalmente fermentadas en el intestino grueso, pudiendo ejercer efectos fisiológicos importantes tanto a nivel local como sistémico (Dahl y Stewart, 2015; Guillon y Champ, 2002). A diferencia de la fibra soluble, la fibra insoluble (celulosa, lignina y algunas fracciones de hemicelulosa) se caracteriza por sufrir una menor fermentación intestinal, siendo excretada mayoritariamente en heces, regulando así el tránsito intestinal (Ros y Periago, 2005). Por último, es destacable el elevado contenido de oligosacáridos de la familia rafinosa presente en legumbres que, junto con otros componentes indigestibles, son responsables de producir flatulencia. No obstante, estudios recientes sugieren un papel importante como compuestos prebióticos emergentes con capacidad para modular la microbiota intestinal (Corzo et al., 2015).

\subsection{Lípidos}

El contenido de lípidos totales en las legumbres es bajo, especialmente en judías y lentejas, donde se mantienen en valores del 1-2\%. Mención especial han de recibir el garbanzo, con niveles del 7-8 \% de contenido graso, y las oleaginosas, entre las que destacan la soja con un contenido lipídico superior al 20\% (Ros y Periago, 2005). Los lípidos constituyen un grupo heterogéneo que incluyen ácidos grasos libre, di- y triglicéridos, fosfolípidos, esteroles, glucolípidos y lipoproteínas. Aunque las proteaginosas no tienen especial interés como fuente de ácidos grasos en la dieta, es interesante destacar que su fracción grasa se caracteriza por presentar un elevado contenido en ácidos grasos monoinsaturados, en particular ácido oleico, y poliinsaturados, como el linoleico y el $\alpha$-linolénico. Es por ello que la Guía Alimentaria de Canadá (Government of Canada, 2007) sugiere el consumo regular de lentejas y judías como alternativas a la carne con objeto de disminuir la ingesta de grasa saturadas.

\subsection{Vitaminas y minerales}

Las legumbres se consideran una fuente de vitaminas hidrosolubles en la dieta. De manera general, podemos afirmar que el contenido de tiamina es del mismo orden o superior del que poseen las semillas de cereales; el aporte de niacina se encuentra en el intervalo 2-3 $\mathrm{mg} / 100 \mathrm{~g}$ (Ros y Periago, 2005). Respecto al ácido fólico, vitamina de vital importancia para prevenir la anemia y los defectos del tubo neural durante la gestación, se ha estimado que el consumo de $100 \mathrm{~g}$ de judías, garbanzos o lentejas cubre prácticamente la totalidad de las necesidades diarias de este nutriente para un adulto sano (400 $\mu \mathrm{g}$ ) (Ros y Periago, 2005). Para preservar su contenido en folato, se recomiendan tiempos de remojo prolongados que favorezcan un cocinado más rápido, ya que el tratamiento térmico es la operación que desencadena más pérdidas (Hoppner y Lampi, 1993).

Las legumbres constituyen una excelente fuente de minerales, en particular hierro, zinc y calcio (Tabla 1) (Campos-Vega, Loarca-Piña y Oomah, 2010). Se han observado niveles elevados de zinc en altramuz, lenteja y garbanzo (24- $176 \mu \mathrm{g} / \mathrm{g}$ de semilla seca) mientras que los niveles más elevados de calcio se han detectado en altramuz, habas, judías y garbanzos (562- $4065 \mu \mathrm{g} / \mathrm{g}$ de semilla seca) (Sparvoli, Bollini y Comibelli, 2015). Los niveles de hierro más elevados lo presentan las judías, habas y lentejas (55-280 $\mu \mathrm{g} / \mathrm{g}$ de semilla seca). A pesar de la riqueza en minerales de las legumbres, se ha de tener en cuenta que se trata de micronutrientes de baja disponibilidad ya que se 
Tabla 1. Composición nutricional media de las legumbres de mayor interés de consumo humano(1)

\begin{tabular}{|c|c|c|c|c|c|c|c|c|c|c|}
\hline \multirow{2}{*}{ Componentes } & \multirow{2}{*}{ Unidades } & \multicolumn{3}{|c|}{ Judías } & \multirow{2}{*}{ Lentejas } & \multirow{2}{*}{ Garbanzos } & \multirow{2}{*}{$\begin{array}{c}\text { Guisantes } \\
\text { verdes }\end{array}$} & \multirow{2}{*}{ Soja } & \multirow{2}{*}{ Habas } & \multirow{2}{*}{ Altramuz } \\
\hline & & Negras & Blancas & Pintas & & & & & & \\
\hline Agua & $\mathrm{g}$ & 11.02 & 12.10 & 11.33 & 8.26 & 7.68 & 78.86 & 8.54 & 10.98 & 10.44 \\
\hline Energía & kcal & 341 & 337 & 347 & 352 & 378 & 81 & 445 & 341 & 371 \\
\hline Proteína & $\mathrm{g}$ & 21.6 & 22.33 & 21.42 & 24.63 & 20.47 & 5.42 & 36.49 & 26.12 & 36.17 \\
\hline Lípidos totales & $\mathrm{g}$ & 1.42 & 1.50 & 1.23 & 1.06 & 6.04 & 0.40 & 19.94 & 1.53 & 9.74 \\
\hline Carbohidratos & $\mathrm{g}$ & 62.36 & 60.75 & 62.55 & 63.35 & 62.95 & 14.45 & 30.16 & 58.29 & 40.37 \\
\hline \multirow{2}{*}{$\begin{array}{l}\text { Fibra total } \\
\text { Azúcares } \\
\text { totales }\end{array}$} & $\mathrm{g}$ & 15.5 & 15.3 & 15.5 & 10.7 & 12.2 & 5.7 & 9.3 & 25.0 & 18.9 \\
\hline & g & 2.12 & 3.88 & 2.11 & 2.03 & 10.70 & 5.67 & 7.33 & 5.70 & - \\
\hline \multicolumn{11}{|l|}{ Minerales } \\
\hline $\mathrm{Ca}$ & $\mathrm{mg}$ & 123 & 147 & 113 & 35 & 57 & 25 & 277 & 103 & 176 \\
\hline $\mathrm{Fe}$ & $\mathrm{mg}$ & 5.02 & 5.49 & 5.07 & 6.51 & 4.31 & 1.47 & 15.70 & 6.70 & 4.36 \\
\hline $\mathrm{Mg}$ & $\mathrm{mg}$ & 171 & 175 & 176 & 47 & 79 & 33 & 280 & 192 & 198 \\
\hline$P$ & $\mathrm{mg}$ & 352 & 407 & 411 & 281 & 252 & 108 & 704 & 421 & 440 \\
\hline K & $\mathrm{mg}$ & 1483 & 1185 & 1393 & 677 & 718 & 244 & 1797 & 1062 & 1013 \\
\hline $\mathrm{Na}$ & $\mathrm{mg}$ & 5 & 5 & 12 & 6 & 24 & 5 & 2 & 13 & 15 \\
\hline $\mathrm{Zn}$ & $\mathrm{mg}$ & 3.65 & 3.65 & 2.28 & 3.27 & 2.76 & 1.24 & 4.89 & 3.14 & 4.75 \\
\hline \multicolumn{11}{|l|}{ Vitaminas } \\
\hline Tiamina $\left(\mathrm{B}_{1}\right)$ & $\mathrm{mg}$ & 0.900 & 0.775 & 0.713 & 0.873 & 0.477 & 0.266 & 0.874 & 0.555 & 0.640 \\
\hline Riboflavina $\left(\mathrm{B}_{2}\right)$ & $\mathrm{mg}$ & 0.193 & 0.164 & 0.212 & 0.211 & 0.212 & 0.132 & 0.870 & 0.333 & 0.220 \\
\hline Niacina $\left(B_{3}\right)$ & $\mathrm{mg}$ & 1.955 & 2.188 & 1.174 & 2.605 & 1.541 & 2.090 & 1.623 & 2.832 & 2.190 \\
\hline Piridoxina $\left(\mathrm{B}_{6}\right)$ & $\mathrm{mg}$ & 0.286 & 0.428 & 0.474 & 0.540 & 0.535 & 0.169 & 0.377 & 0.366 & 0.357 \\
\hline Ac. ascórbico (C) & $\mathrm{mg}$ & - & - & 6.3 & 4.5 & 4.0 & 40.0 & 6.0 & 1.4 & 4.8 \\
\hline Folatos & $\mu \mathrm{g}$ & 444 & 364 & 525 & 479 & 557 & 65 & 375 & 423 & 355 \\
\hline Filoquinona (K) & $\mu g$ & 5.6 & 2.5 & 5.6 & 5.0 & 9.0 & 24.8 & 47.0 & 9.0 & - \\
\hline \multicolumn{11}{|l|}{ Lípidos } \\
\hline Saturados & $\mathrm{g}$ & 0.366 & 0.170 & 0.235 & 0.154 & 0.603 & 0.071 & 2.884 & 0.254 & 1.156 \\
\hline Monoinsaturados & $\mathrm{g}$ & 0.123 & 0.128 & 0.229 & 0.193 & 1.377 & 0.035 & 4.404 & 0.303 & 3.940 \\
\hline Poliinsaturados & $\mathrm{g}$ & 0.610 & 0.873 & 0.407 & 0.526 & 2.731 & 0.187 & 11.255 & 0.627 & 2.439 \\
\hline Número $\mathrm{NDB}^{(2)}$ & & 16014 & 16037 & 16042 & 16069 & 16056 & 11304 & 16108 & 16052 & 16076 \\
\hline
\end{tabular}

${ }^{(1)}$ Datos obtenidos de USDA National Nutrient Database for Standard Reference Release 28 para 100g de semilla cruda, ligeramente modificada en mayo de 2016. Disponible en: http://ndb.nal.usda.gov/ndb/search

${ }^{(2)}$ Número NDB: código de identificación del alimento en la base de datos.

Los guiones indican cantidades no detectables o datos no proporcionados para ese alimento.

encuentran mayoritariamente formando complejos con el ácido fítico, que constituye el principal inhibidor de la absorción de calcio, hierro y zinc. Algunos compuestos fenólicos existentes en las legumbres, como el ácido tánico, poseen también capacidad quelante de minerales, incidiendo igualmente en el descenso de su biodisponibilidad (Olmedilla Alonso, Farré Rovir, Asensio Vegas y Martín Pedrosa, 2010).

\subsection{Compuestos fitoquímicos}

Las leguminosas poseen un gran número de compuestos bioactivos o fitoquímicos que tradicionalmente se han considerado como factores anti-nutricionales, pero de los que en la actualidad se conoce que pueden tener efectos metabólicos y fisiológicos de interés (Olmedilla et al., 2010). Las legumbres contienen proteínas que son resistentes al proceso digestivo, tales como lectinas, inhibidores de proteasas y proteínas $2 \mathrm{~S}$, las cuales permanecen biológicamente activas en el tracto gastrointestinal. Diversos estudios, realiza- dos fundamentalmente en la décadas de los 80 y 90 , mostraron que algunas de estas proteínas afectaban de manera negativa los parámetros de producción en animales de experimentación, principalmente roedores, argumentándose que podrían ejercer propiedades anti-nutritivas en humanos. En la actualidad, existen numerosas evidencias científicas que demuestran que estas proteínas resistentes a la digestión pueden ejercer efectos beneficiosos para la salud.

Las lectinas o fitohemaglutininas, llamadas así por su capacidad para aglutinar in vitro glóbulos rojos, son glicoproteínas con capacidad de unión a carbohidratos de manera específica y reversible. Las lectinas pueden unirse a azúcares y glicoproteínas situados en la superficie celular del epitelio intestinal, interfiriendo los procesos de digestión y absorción. Investigaciones recientes han demostrado el papel de las lectinas como reguladores hormonales, metabólicos y digestivos así como moduladores del sistema inmune y de la microbiota intestinal (Pusztai, 2008). 
Los inhibidores de proteasas de Bowman-Birk son proteínas con elevado contenido en puentes disulfuro, resistentes al tratamiento térmico y las condiciones extremas del proceso digestivo ( $\mathrm{pH}$ ácido y acción de enzimas proteolíticas). Numerosos estudios han evidenciado el potencial de estas proteínas en la prevención y/o supresión de procesos cancerígenos y desórdenes inflamatorios utilizando un amplio abanico de modelos in vitro e in vivo (Clemente, Sonnante y Domoney, 2011; Utrilla et al., 2015).

Los taninos condensados son compuestos fenólicos solubles en agua y de gran complejidad estructural de los que se conoce su capacidad para formar complejos solubles e insolubles con proteínas (Ros y Periago, 2005). De este modo, la presencia elevada de taninos se ha asociado con la reducción de la biodisponibilidad proteica y también de carbohidratos y lípidos, ya que inhiben las enzimas tripsina, $\alpha$-amilasa y lipasa (Suárez-Martínez et al., 2016).

La fibra (soluble e insoluble), el almidón resistente y distintos oligosacáridos son responsables del bajo índice glicémico de las legumbres, demostrándose sus efectos saciantes, hipocolesterolémicos y anticancerígenos (Morel et al., 2015; Vergara-Castañeda et al. 2010). Finalmente, las saponinas están presentes en diversas legumbres -altramuz, lentejas, judías, garbanzos, guisantes y soja-, aunque su estructura detallada no está bien establecida. Estudios epidemiológicos sugieren que las saponinas de legumbres pueden jugar un papel protector frente al desarrollo de procesos tumorales (Chang, Yu, Lin, Wang y Tsai, 2006). Otros autores han sugerido también que pueden tener una acción hipolipidémica mediada por la formación de un complejo insoluble con el colesterol en el lumen intestinal que prevendría su absorción (Rochfort y Panozzo, 2007).

\section{EVIDENCIAS CIENTÍFICAS DE LAS PROPIEDADES BENE- FICIOSAS DE LAS LEGUMBRES EN LA SALUD HUMANA}

Estudios epidemiológicos, estudios in vitro e in vivo utilizando modelos animales así como estudios de intervención en humanos han demostrado que la ingesta de legumbres proporciona beneficios en salud cardiovascular, control de peso, síndrome metabólico, salud gastrointestinal y diabetes tipo-2. Los efectos fisiológicos de la ingesta de diferentes especies de legumbres pueden variar significativamente, incluso a nivel de variedad vegetal, debido a las diferencias en su composición en polisacáridos, cantidad y variedad en fibra alimentaria y almidón, composición en proteínas así como a la abundancia y tipo de determi- nados compuestos fitoquímicos. El consumo de estos últimos puede proporcionar beneficios para la salud protegiendo contra enfermedades coronarias, presión sanguínea elevada e inflamación, entre otras. El descubrimiento de los efectos sinérgicos o antagonistas de estos compuestos fitoquímicos provenientes de legumbres en la alimentación diaria, sus interacciones y mecanismos de acción necesitan ser investigados con mayor profundidad.

\subsection{Salud cardiovascular}

Las enfermedades cardiovasculares son una de las principales causas de mortalidad a nivel mundial (30 $\%$ del total), siendo su incidencia mucho más elevada en países desarrollados. Aunque los factores hereditarios son determinantes en el desarrollo de estas patologías, existen evidencias científicas que atribuyen al estilo de vida y, en particular a la dieta, un papel relevante. Se ha demostrado que un consumo de legumbres de cuatro raciones a la semana disminuye un $22 \%$ el riesgo de sufrir enfermedades cardiovasculares frente a individuos cuya ingesta es inferior a una ración (Bazzano, Thompson, Tess, Nguyen y Winham, 2011).

Son numerosos los estudios que han demostrado el efecto beneficioso de la ingesta de proteínas de soja en la reducción de los niveles de colesterol. Dichos estudios, dieron lugar a que la FDA aprobara el atribuir a las proteínas de la soja propiedades beneficiosas para la salud cardiovascular; en concreto, los productos deben contener al menos $6.25 \mathrm{~g}$ de proteínas de soja para poder incluir alegaciones de salud en su etiquetado (FDA, 1999). Un estudio reciente ha demostrado que tanto las proteínas procedentes del altramuz como las de guisante son efectivas a la hora de reducir los niveles de colesterol en sangre, siendo más eficaces las primeras (Sirtori et al., 2012). Estudios realizados en individuos mayores de 50 años mostraron que la ingesta moderada de legumbres disminuye hasta un $6 \%$ los niveles totales de colesterol, reduciendo así el riesgo de desarrollar enfermedades cardiovasculares (Abeysekara, Chilibeck, Vatanparast y Zello, 2012); no obstante, los mecanismos de acción responsables de estos efectos necesitan ser estudiados en mayor profundidad.

El consumo de legumbres aporta cantidades significativas de fibra y compuestos bioactivos tales como esteroles, saponinas y oligosacáridos, los cuales ayudan a disminuir los niveles de lípidos totales y colesterol en personas obesas (Pittaway, Robertson y Ball, 2008). Estudios de intervención demuestran que la ingesta de garbanzos germinados son eficaces en el des- 
censo de los niveles de colesterol en sangre (Jukanti, Gaur, Gowda y Chibbar, 2012). Este hecho se ha atribuido a la síntesis de compuestos bioactivos durante el proceso de germinación de las semillas, incluidas las saponinas, las cuales forman un complejo insoluble con el colesterol y previenen su absorción en el intestino delgado, ejerciendo un efecto hipocolesterolémico (Serventi et al., 2013). Además, se ha demostrado que aquellos individuos que ingieren fibra de guisante en sus dietas muestran una disminución en los niveles postprandiales de triglicéridos respecto a aquellos que consumen una dieta baja en fibra (Sandstrom, Hansen y Sorensen, 1994).

Uno de los factores de riesgos para sufrir una enfermedad cardiovascular es la hipertensión. Estudios clínicos han demostrado que ciertas proteínas vegetales son efectivas en reducir la presión arterial (Martínez-Maqueda, Miralles, Recio y Hernández-Ledesma, 2012). Así, se ha observado que la ingesta de proteínas procedentes del altramuz blanco (L. albus) reduce tanto la presión sanguínea (sistólica y diastólica) como los niveles de colesterol LDL en sangre (Bähr, Fechner, Krämer, Kiehntopf y Jahreis, 2013). En un estudio con individuos que presentaban moderada hipertensión, el consumo diario de $35 \mathrm{~g}$ de proteína de altramuz redujo la presión sanguínea de manera significativa (Nowicka, Klosiewicz-Latoszek, Sirtori, Arnoldi y Naruszewicz, 2006). Otro ensayo de intervención utilizando aislados proteicos de altramuz, garbanzo, lenteja y judía estableció una reducción de la presión arterial como consecuencia de la liberación de péptidos hipotensivos liberados durante el proceso digestivo (Boschin, Scigliuolo, Resta y Arnoldi, 2014); estos autores mostraron que los más eficaces eran aquellos provenientes de altramuz.

Finalmente, es necesario considerar el posible efecto positivo que ejerce el consumo de legumbres sobre lo que se conoce como síndrome metabólico. El síndrome metabólico engloba un grupo de condiciones metabólicas asociadas con riesgos de padecer enfermedades cardiovasculares, elevados niveles de triglicéridos y colesterol LDL, bajos niveles de colesterol $\mathrm{HDL}$, mayor volumen de tejido adiposo, niveles elevados de glucosa en sangre y presión sanguínea elevada. Se ha observado que la ingesta de judías blancas pueden tener un efecto protector frente al síndrome metabólico gracias al retraso en la utilización de glucosa como combustible celular, sustituyéndola por el uso de lípidos y controlando el apetito a través del incremento de la saciedad (Anderson y Major, 2002). Los resultados obtenidos en dos estudios meta-análisis mostraron los beneficios del consumo de legumbres (2-5 raciones de legumbres durante 3-12 semanas) sobre determinados factores de riesgo del síndrome metabólico (Bazzano et al., 2011; Sievenpiper et al., 2009). Por otro lado, se ha demostrado el efecto beneficioso del consumo de soja sobre factores relativos al síndrome metabólico; así, se ha atribuido a la ingesta de oligosacáridos presentes en soja una reducción de niveles anormales de glucosa y lípidos totales en sangre así como una mejora en el estrés oxidativo celular (Chang, Kim, Kim y Lee, 2008). Recientemente, se ha mostrado que un cambio en los hábitos alimenticios de pacientes con diabetes tipo-2, sustituyendo el consumo de carnes rojas por legumbres, mejora significativamente algunos de los parámetros relativos al síndrome metabólico, tales como el perfil lipídico y el control glicémico (Hosseinpour-Niazi, Mirmiran, Hedayati y Azizi, 2015).

\subsection{Obesidad y sobrepeso}

El sobrepeso y la obesidad son considerados como uno de los problemas con mayor impacto sobre la salud a los que se enfrenta nuestra sociedad occidental. La prevalencia de la obesidad ha aumentado exponencialmente, sobre todo en los países industrializados, teniendo a la diabetes, hipertensión y enfermedades cardiovasculares como sus consecuencias más directas. Recientemente, se ha demostrado la importancia de las legumbres en dietas hipocalóricas, reduciendo el nivel de marcadores pro-inflamatorios y mejorando ciertas características metabólicas en individuos con sobrepeso y obesidad (Abete, Parra y Martínez, 2009; Hermsdorff, Zullet, Abete y Martínez, 2011); no obstante, existen escasas evidencias científicas sobre el papel que juegan en parámetros tales como control del peso, índice de masa corporal (IMC) o circunferencia abdominal. La ingesta de alimentos ricos en fibra ayuda a alcanzar un estado de saciedad más rápidamente, siendo esta situación más duradera en el tiempo (Dahl, Foster y Tyler, 2012). En este sentido, los niveles elevados de almidón resistente y fibra dietética presentes en las legumbres pueden ejercer un efecto en el control del apetito, aumentando la sensación de saciedad. Lunde, Hjellset, Holmboe-Ottesen y Hostmark (2011) demostraron que panes enriquecidos con fibra de guisante incrementan el periodo de saciedad, en comparación a lo que el individuo percibe tras la ingesta de un pan normal. Por otro lado, se ha demostrado que el consumo de garbanzo, considerado como un alimento con bajo índice glicémico, incrementa los niveles de secreción de colecistoquinina, hormona intestinal que regula la sensación de 
apetito (McCrory, Hamaker, Lovejoy y Eichelsdoerfer, 2010). En un estudio en el que participaron más de 8000 adultos, se asoció el consumo de judías dentro de una dieta de restricción calórica en pacientes con sobrepeso con un descenso significativo en el riesgo a padecer obesidad (Papanikolaou y Fulgoni, 2008).

\subsection{Salud gastrointestinal}

La microbiota intestinal juega un papel fundamental en la salud y bienestar del individuo. Las bacterias intestinales facilitan nutrientes y energía al hospedador, fermentando y absorbiendo compuestos no digeribles de la dieta y liberando distintos compuestos, tales como vitaminas esenciales y ácidos grasos de cadena corta que influyen en la fisiología del individuo a nivel local y sistémico (Flint, Scott, Louis y Duncan, 2012). La disponibilidad de nutrientes es crítica en la modulación de la composición y actividad metabólica de la microbiota (Roberfroid et al., 2010). Una dieta enriquecida en frutas y fibra determina una mayor diversidad microbiana y puede ejercer un efecto positivo en la salud intestinal (Simpson y Campbell, 2015).

Estudios in vitro han demostrado que las enzimas digestivas son incapaces de hidrolizar oligosacáridos de la familia rafinosa por carecer de actividad $\alpha$-galactosidasa, pudiendo alcanzar el intestino grueso donde son degradados por la microbiota intestinal; su fermentación favorecen el crecimiento de bifidobacterias (Espinosa-Martos y Rupérez, 2006). En un estudio reciente llevado a cabo en adultos con sobrepeso, se observó que la ingesta de fibra soluble de legumbres durante un periodo de 14 días redujo el apetito, la ingesta de alimentos así como la presencia de marcadores pro-inflamatorios, siendo dicho efecto dosis-dependiente (Morel et al., 2015).

El cáncer colorectal (CCR) constituye una de las principales causas de muerte por cáncer a nivel mundial. CCR es una enfermedad compleja y heterogénea que resulta de una combinación de factores hereditarios, ambientales, de estilo de vida y dietético. La intervención nutricional y/o modificación de la dieta son herramientas claves en la prevención y reducción en la incidencia del CCR. El hecho de que determinados componentes de la dieta tengan propiedades quimiopreventivas tiene notables implicaciones en salud pública, debiéndose promover el consumo de estos compuestos bioactivos en la población, una vez conocidas las bases científicas de sus efectos beneficiosos en salud. Los efectos anti-cancerígenos de las legumbres han sido evaluados. Aunque la evidencia científica es limitada, existen varios estudios que sugieren que la ingesta de legumbres junto con el de frutas frecas y verduras pueden ejercer un efecto protector. En un estudio caso-control, Aune, De Stefani, Ronco, Boffetta, Deneo-Pellegrini, Acosta y Mendilaharsu, (2009) sugirieron que la ingesta de legumbres necesaria para ejercer un efecto protector frente a distintos tipos de cáncer, incluído CCR, es de dos pequeñas porciones, equivalentes a 100 gramos de legumbres por semana, pudiendo ser fácilmente llevado a la práctica por la población. Un meta-análisis de tres estudios cohorte y once casos-estudio sugieren una correlación negativa entre los niveles de ingesta de legumbres y el riesgo a padecer CCR (Aune et al., 2009).

La desregulación de ciertas actividades proteolíticas de serín proteasas durante los procesos inflamatorios y/o cancerígenos dentro del tracto gastrointestinal ha impulsado a la comunidad científica a investigar el potencial de inhibidores, naturales y/o sintéticos, que modulen dichas actividades. Así, los inhibidores de serín proteasas de la familia de Bowman-Birk (BBI), presentes en semillas de leguminosas tales como la soja, la lenteja, el guisante y el garbanzo, están siendo evaluados como agentes quimiopreventivos colorectales (Clemente, 2014). Cantidades fisiológicamente relevantes de estos inhibidores de serín proteasas del tipo tripsina y quimotripsina alcanzan el intestino grueso en forma activa debido a su extraordinaria resistencia a condiciones extremas $(\mathrm{pH}$ ácido, acción de enzimas digestivas y actividad metabólica/proteolítica de la microbiota intestinal) (Clemente, Jiménez, Marín-Manzano y Rubio, 2008; Marín-Manzano, 2009). Recientemente se ha demostrado que los BBI de guisante ejercen propiedades anti-inflamatorias en un modelo de colitis ulcerosa inducida en ratón (Utrilla et al., 2015). Asimismo, el tratamiento con isoformas BBI prodecentes de distintas fuentes de leguminosas (guisante, lenteja y soja) da lugar a un descenso dosis- y tiempo-dependiente en la proliferación de células procedentes de adenocarcinoma colorectal humano (Clemente, Moreno, Marín-Manzano, Jiménez y Domoney, 2010); este efecto está asociado a su capacidad intrínseca de inhibir serín proteasas (Clemente, Marín-Manzano, Jiménez, Arques y Domoney, 2012). En este sentido, serin proteasas del tipo tripsina $y$ quimotripsina han sido consideradas posibles dianas terapéuticas para $\mathrm{BBI}$ en estadíos primarios de cáncer colorectal; sin embargo, sus dianas terapéuticas específicas no han sido determinadas. 


\subsection{Actividad antioxidante}

Según la hipótesis antioxidante, vitaminas y compuestos fitoquímicos de la matriz alimentaria pueden jugar un papel importante en la etiología de enfermedades crónicas, en especial las cardiovasculares, debido a que se trata de herramientas fundamentales en la lucha contra las especies reactivas de oxígeno (ERO) originadas durante el metabolismo de los sistemas biológicos (Blomhoff, 2005). Aunque el organismo no está indefenso frente a la acción de ERO, los mecanismos enzimáticos y endógenos pueden no ser suficiente para neutralizar los procesos de oxidación; por ello, es primordial el aporte de antioxidantes en la dieta (Saura-Calixto y Goñi, 2005). Estudios recientes catalogan a las legumbres como fuentes de antioxidantes naturales. En lentejas, judías, guisantes y garbanzos los compuestos más abundantes son tanto de tipo flavonoide (flavonoles como quercetina; flavanoles como las proantocianidinas; isoflavonas como daidzeína y genisteína; etc.), como no flavonoideo (ácidos hidroxicinámicos e hidroxibenzoicos y derivados) (Ros y Periago, 2005); en habas predomina el flavonol kenferol (Neugart, Ron y Schreiner, 2015) y en la soja y sus derivados las isoflavonas genisteína, daidzeína y glicitina 7-O- $\beta$-glucósido, y el cumesterol (Ros y Periago, 2005). Es evidente que el perfil antioxidante de las legumbres de consumo estará determinado de una parte por el contenido natural en compuestos fenólicos de la variedad y, de otra, por los efectos que los tratamientos tecnológicos y culinarios puedan tener sobre la conservación de estos compuestos en el producto final. Así, se ha establecido que la cantidad de glucósidos de flavanoles puede incrementarse de forma significativa tras el cocinado de los garbanzos o la germinación de los guisantes, lentejas y judías (Matthews, 1989). Por su parte, en la soja y sus derivados, la presencia de genisteína decrece a medida que el producto sufre un mayor procesado tecnológico. Sin embargo, el cumesterol, que está presente en muy bajas concentraciones en la soja cruda y derivados, puede aumentar su contenido entre 8 y 200 veces tras el proceso de germinación (Ros y Periago, 2005).

\section{5. Índice glicémico y resistencia a insulina}

En la actualidad, los cambios en los hábitos alimentarios son una estrategia fundamental en las terapias antidiabéticas. Así, investigaciones recientes apuntan a que las leguminosas pueden jugar un papel importante en la prevención y tratamiento de diabetes meIlitus (Campos-Vega et al., 2010; Jenkins et al., 2012). Estas nuevas terapias dietéticas se basan en estudios epidemiológicos que han demostrado que el consumo de tres o más porciones por semana de legumbres reduce entre un 20-35\% el riesgo de padecer diabetes (Kirpitch y Maryniuk, 2011). Diversos estudios de corta duración han demostrado que el consumo de legumbres en adultos diabéticos y no-diabéticos reduce la elevación de los niveles de glucosa postprandial cuando se compara con la ingesta de alimentos ricos en almidón (Venn y Mann, 2004).

El garbanzo es una de las legumbres con elevados niveles de almidón resistente así como de amilosa; ambos componentes reducen la disponibilidad de glucosa en sangre disminuyendo la demanda de insulina (Osorio-Díaz, Agama-Acevedo, Mendoza-Vinalay, Tovar y Bello-Pérez, 2008). Si el consumo de este tipo de polisacáridos de lenta liberación de glucosa es persistente en el tiempo, se consigue, a medio plazo, una mayor tolerancia a la glucosa y mejora la resistencia a la insulina (Nestel, Ceun y Chronopoulos, 2004). En definitiva, el consumo de garbanzo disminuye el índice glucémico y la respuesta insulinémica, conduciendo a un control más estrecho de la diabetes tipo 2, que cursará así de forma menos severa. Por otro lado, estudios realizados empleando proteínas y fibra de soja han evidenciado su utilidad para disminuir la glucemia y mejorar la respuesta eficaz a la insulina (Villegas et al., 2008), siendo atribuido no sólo a la fracción proteica sino también a la presencia de isoflavonas. Las isoflavonas mayoritarias en la soja son la genistina, daidzeina y glicitina, de las que se conoce su potente efecto como inhibidores de la acción de la $\alpha$-glucosidasa, una enzima importante en la digestión de carbohidratos (Lee y Lee, 2001). A este efecto inhibitorio digestivo se suma la baja digestibilidad de los carbohidratos de la soja, debido a la presencia de fibra soluble y al elevado contenido en amilosa y almidón resistente a la digestión. Todo ello, junto con la abundancia de ácidos grasos de cadena corta, previene un elevado nivel de glucosa en sangre, resultando en una respuesta reducida de insulina (Kwon, Daily, Kim y Park, 2010).

En los últimos años, se ha prestado gran atención al altramuz en relación a sus propiedades anti-diabéticas. Varias especies del genero Lupinus han demostrado su potencial como agentes hipoglucémicos, tanto en estrategias de prevención como en el tratamiento de esta patología. Estas conclusiones se han extraído de ensayos basados en el consumo de derivados de cereales, como el pan y la pasta, que se suplementaban con harina procedente de semillas de altramuz (Capraro et al., 2014). La responsable de 
estos efectos beneficiosos es una proteína minoritaria (4-5\%), la conglutina gamma. Esta proteína, procedente de altramuz blanco (L. albus), ha sido evaluada tanto en estudios in vitro como in vivo demostrándose que, tras su administración oral, los niveles de glucosa en sangre se reducen de forma dosis-dependiente, al igual que los niveles de insulina en sangre (Bertoglio et al., 2012). El mecanismo de acción de esta proteína antidiabética parece deberse a la activación de una de las vías de señalización celular de la insulina, ejerciendo un efecto mimético al de esta hormona (Terruzzi et al., 2011).

\section{HÁBITOS Y RECOMENDACIONES DE CONSUMO DE LEGUMBRES}

Las legumbres junto a cereales, verduras, frutas frescas y hortalizas juegan un papel fundamental en el perfil de lo que conocemos como dieta mediterránea. En este contexto, España es uno de los países europeos cuyos ciudadanos consumen más legumbres (FAOSTAT, 2015). No obstante, los datos de consumo de los hogares españoles recogidos en el Panel de Consumo Alimentario del Ministerio de Agricultura, Alimentación y Medio Ambiente (MAGRAMA, 2015) señalan que, en las últimas décadas, ha habido un descenso muy significativo, superior al $60 \%$, en el consumo de legumbres (Figura 1). A comienzos de los 90 , el consumo de legumbres se situaba en valores de 7,4 kg por persona/año, siendo utilizadas en la cocina tradicional casi a diario (Varela, 2014). En el periodo 2000-2008, se observó un descenso pronunciado en el consumo de legumbres, con valores en torno a 4.4-4.9 kg por persona/año, siendo este consumo independiente del status socioeconómico de los individuos y del tamaño de la población en la que residían (Valoración Nutricional 2012). En 2014, el volumen de legumbres consumidas en España fue de 140.888 Tm, con un consumo anual per cápita de 3.1 kilos (MAGRAMA, 2015). En la actualidad, los garbanzos (1.25 kg/cápita), las alubias (0.93 kg/cápita) y las lentejas (0.93 kg/cápita) son las legumbres más consumidas en los hogares españoles. Existen evidencias de una clara evolución de nuestra dieta hacia patrones más occidentalizados, aunque ésta continua siendo rica en productos vegetales (fundamentalmente, verduras, frutas y hortalizas) y menos en legumbres y cereales (Varela, 2014). Este descenso significativo en el consumo de legumbres puede ser debido a numerosos factores, incluidos los cambios en los modelos socioeconómicos, modelos de producción y estilos de vida de los ciudadanos.
En la actualidad, científicos e industria alimentaria trabajan en colaboración para diseñar nuevos productos alimenticios basados en legumbres al objeto de restaurar el consumo de esta importante fuente de proteínas, minerales, vitaminas y fibra en la dieta. Precisamente, esta composición las convierte en ingredientes para la mejora de la calidad nutricional de alimentos, confiriéndoles menor índice glicémico, buen perfil antioxidante así como capacidad de retención de agua y de absorción de grasas. Además, están libres de gluten, lo que potencia aún más sus aplicaciones para la elaboración de productos destinados a colectivos especiales. Así, las harinas de garbanzo son empleadas en la elaboración de pan libre de gluten, mejorando sus características nutricionales y organolépticas. Además, las harinas de legumbres son utilizadas en el diseño de nuevos snacks saludables, por sí solas o en combinación con harinas de cereales, utilizándose como estrategia para fortificar el producto final, especialmente en su perfil de aminoácidos y contenido mineral (Rababah et al., 2012). Otras aplicaciones son la fabricación de bollería y pastas fortificadas y aptas para celíacos, sustitutos de la cuajada y la leche destinados a intolerantes a la lactosa, sopas, pasta y productos enlatados, entre otros (Boye, Zare y Pletch, 2010). A través de estas innovaciones se insta al consumo de dietas más sanas y nutritivas e, indirectamente, a incrementar la ingesta de legumbres, debido a las ventajas que ofrecen los nuevos alimentos frente a los tradicionales (Vaz Patto et al., 2015).

En los últimos años, y con objeto de fomentar una alimentación saludable, se han publicado recomendaciones sobre la ingesta de legumbres por parte de distintas Administraciones y Agencias, de ámbito nacional e internacional. Generalmente, las recomendaciones se suelen expresar en términos de raciones/ semana, definiéndose ración como la cantidad equivalente a 60-80 gramos de legumbres en crudo o 150200 gramos cocidas. Atendiendo a las recomendaciones de la Fundación Española de la Nutrición (www. fen.org.es), se aconseja un consumo semanal de 3-4 raciones de legumbres (SENC y SEMFYC, 2007), siendo el consumo actual de la población adulta española de 1.4 raciones por semana. Otras agencias internacionales han publicado recomendaciones relativas al consumo de legumbres como elementos claves de una dieta saludable. Así, el Departamento de Agricultura de Estados Unidos (USDA, 2011) y el Comité de Nutrición de Legumbres y Cereales de Australia (http:// www.glnc.org.au) recomiendan consumir al menos 2-3 raciones de legumbres a la semana con objeto de reducir el riesgo de enfermedades crónicas como la 
Figura 1. Evolución del consumo anual de legumbres en la población española (1981-2014)

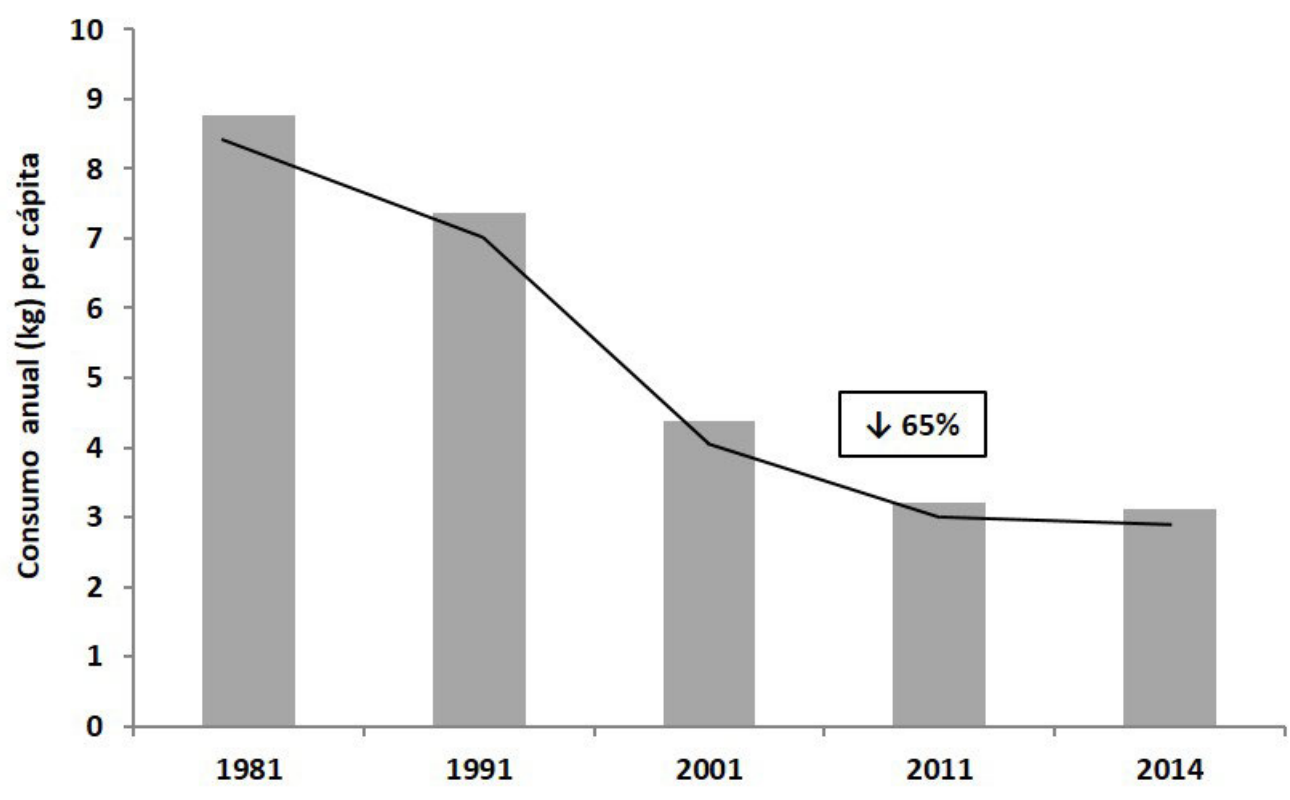

Fuentes: Varela, 2014 y series estadísticas del MAGRAMA.

diabetes, cáncer y enfermedades cardiovasculares; en ambos países, el consumo de leguminosas es inferior a las recomendaciones establecidas (Mitchell, Lawrence, Hartman y Curran, 2009; Australian dietary guidelines, 2013). Por otro lado, la Asociación Americana del Corazón recomienda consumir al menos 4 raciones a la semana de legumbres, frutos secos y semillas para una dieta standard de 2000 Kcal (www. heart.org). Sin duda, el cambio de tendencia requerirá de políticas de promoción de dieta saludable para la población española donde las legumbres deben jugar un papel muy importante.

\section{BIBLIOGRAFÍA}

Abete, I., Parra, D. y Martínez, J. A. (2009). Legume-, Fish-, or HighProtein-Based Hypocaloric Diets: Effects on Weight Loss and Mitochondrial Oxidation in Obese Men. Journal of Medicinal Food, 12, 1, pp. 100-108. http://doi.org/10.1089/jmf.2007.0700

Abeysekara, S., Chilibeck, P. D., Vatanparast, H. y Zello, G. A. (2012). A pulse-based diet is effective for reducing total and LDL-cholesterol in older adults. British Journal of Nutrition, 108, S1, S103S110. http://doi.org/10.1017/S0007114512000748

Anderson, J. W. y Major, A. W. (2002). Pulses and lipaemia, shortand long-term effect: Potential in the prevention of cardiovascular disease. British Journal of Nutrition, 88, S263-S271. http:// doi.org/10.1079/BJN.2002716

\section{AGRADECIMIENTOS}

Alfonso Clemente agradece la financiación a través de los proyectos CSIC Intramural 201540E085 y 201640E039. Cristina Delgado-Andrade y Jose Carlos Jiménez-López agradecen al Ministerio de Economía y Competitividad del Gobierno de España por financiar los proyectos RYC-2008-02450 y RYC-2014-16536 (Programa de investigación Ramón y Cajal), respectivamente. Jose Carlos Jiménez-López agradece la financiación al programa de Investigación Europeo MARIE CURIE (FP7-PEOPLE-2011-IOF) a través del proyecto PIOF-GA-2011-301550.

Aune, D., De Stefani, E., Ronco, A., Boffetta, P., Deneo-Pellegrini, H., Acosta, G. y Mendilaharsu, M. (2009). Legume intake and the risk of cancer: a multisite case-control study in Uruguay. Cancer Causes y Control, 20, 9, pp. 1605-1615. http://doi.org/10.1007/ s10552-009-9406-z

Australian dietary guidelines 2013. Eat for Health. [En línea]. [Fecha de consulta: 18 de febrero de 2016]. Disponible en: https:// www.eatforhealth.gov.au/sites/default/files/files/the_guidelines/n55_australian_dietary_guidelines.pdf

Bähr, M., Fechner, A., Krämer, J., Kiehntopf, M. y Jahreis, G. (2013). Lupin protein positively affects plasma LDL cholesterol and LDL:HDL cholesterol ratio in hypercholesterolemic adults af- 
ter four weeks of supplementation: a randomized, controlled crossover study. Nutrition Journal, 12, 1, 107. http://doi. org/10.1186/1475-2891-12-107

Bazzano, L. A., Thompson, A. M., Tees, M. T., Nguyen, C. H. y Winham, D. M. (2011). Non-soy legume consumption lowers cholesterol levels: A meta-analysis of randomized controlled trials. Nutrition, Metabolism and Cardiovascular Diseases, 21, 2, pp. 94-103. http://doi.org/10.1016/j.numecd.2009.08.012

Bertoglio, J. C., Calvo, M. A., Hancke, J. L., Burgos, R. A., Riva, A., Morazzoni, P. y Duranti, M. (2011). Hypoglycemic effect of lupin seed $\gamma$-conglutin in experimental animals and healthy human subjects. Fitoterapia, 82, 7, pp. 933-938. http://doi. org/10.1016/j.fitote.2011.05.007

Blomhoff, R. (2005). Dietary antioxidants and cardiovascular disease. Current Opinion in Lipidology, 16, 1, 47-54. http://doi. org/10.1097/00041433-200502000-00009

Boschin, G., Scigliuolo, G. M., Resta, D. y Arnoldi, A. (2014). ACEinhibitory activity of enzymatic protein hydrolysates from lupin and other legumes. Food Chemistry, 145, pp. 34-40. http://doi. org/10.1016/j.foodchem.2013.07.076

Boye, J., Zare, F. y Pletch, A. (2010). Pulse proteins: Processing, characterization, functional properties and applications in food and feed. Food Research International, 43, 2, pp. 414-431. http:// doi.org/10.1016/j.foodres.2009.09.003

Campos-Vega, R., Loarca-Piña, G. y Oomah, B. D. (2010). Minor components of pulses and their potential impact on human health. Food Research International, 43, 2, pp. 461-482. http:// doi.org/10.1016/j.foodres.2009.09.004

Capraro, J., Magni, C., Scarafoni, A., Caramanico, R., Rossi, F., Morlacchini, M. y Duranti, M. (2014). Pasta supplemented with isolated lupin protein fractions reduces body weight gain and food intake of rats and decreases plasma glucose concentration upon glucose overload trial. Food y Function, 5, 2, pp. 375-380. http:// doi.org/10.1039/c3fo60583c

Chang, J. H., Kim, M. S., Kim, T. W. y Lee, S. S. (2008). Effects of soybean supplementation on blood glucose, plasma lipid levels, and erythrocyte antioxidant enzyme activity in type 2 diabetes mellitus patients. Nutrition Research and Practice, 2, 3, pp. 152157. http://doi.org/10.4162/nrp.2008.2.3.152

Chang, W.-W., Yu, C.-Y., Lin, T.-W., Wang, P.-H. y Tsai, Y.-C. (2006). Soyasaponin I decreases the expression of $\alpha 2,3$-linked sialic acid on the cell surface and suppresses the metastatic potential of B16F10 melanoma cells. Biochemical and Biophysical Research Communications, 341, 2, pp. 614-619. http://doi.org/10.1016/j. bbrc.2005.12.216

Clemente, A. (2000). Enzymatic protein hydrolysates in human nutrition. Trends in Food Science y Technology, 11, 7, pp. 254-262. http://doi.org/10.1016/S0924-2244(01)00007-3

Clemente, A. (2014). Bowman-Birk inhibitors from legumes as colorectal chemopreventive agents. World Journal of Gastroenterology, 20, 30, 10305-10315. http://doi.org/10.3748/wjg.v20. i30.10305

Clemente, A., Marín-Manzano, M. C., Jiménez, E., Arques, M. C. y Domoney, C. (2012). The anti-proliferative effect of TI1B, a major Bowman-Birk isoinhibitor from pea (Pisum sativum L.), on HT29 colon cancer cells is mediated through protease inhibition.
British Journal of Nutrition, 108, Supplement S1, S135-S144. http://doi.org/10.1017/S000711451200075X

Clemente, A., Gee, J. M., Johnson, I. T., MacKenzie, D. A. y Domoney, C. (2005). Pea (Pisum sativum L.) Protease Inhibitors from the Bowman-Birk Class Influence the Growth of Human Colorectal Adenocarcinoma HT29 Cells in Vitro. Journal of Agricultural and Food Chemistry, 53, 23, 8979-8986. http://doi.org/10.1021/jf051528w

Clemente, A., Jiménez, E., Marín-Manzano, M. C. y Rubio, L. A. (2008). Active Bowman-Birk inhibitors survive gastrointestinal digestion at the terminal ileum of pigs fed chickpea-based diets. Journal of the Science of Food and Agriculture, 88, 3, pp. 513-521. http://doi.org/10.1002/jsfa.3115

Clemente, A., Moreno, F. J., Marín-Manzano, M. del C., Jiménez, E. y Domoney, C. (2010). The cytotoxic effect of Bowman-Birk isoinhibitors, IBB1 and IBBD2, from soybean (Glycine max) on HT29 human colorectal cancer cells is related to their intrinsic ability to inhibit serine proteases. Molecular Nutrition y Food Research, 54, 3, pp. 396-405. http://doi.org/10.1002/mnfr.200900122

Clemente, A., Sonnante, G. y Domoney, C. (2011). Bowman-Birk Inhibitors from Legumes and Human Gastrointestinal Health: Current Status and Perspectives. Current Protein y Peptide Science, 12, 5, pp. 358-373.

Corzo, N., Alonso, J. L., Azpiroz, F., Calvo, M. A., Cirici, M., Leis, R., Lombó, F., Mateos-Aparicio, I., Plou, F. J., Ruas-Madiedo, P., Ruperez, P., Redondo-Cuenca, A., Sanz, M. L. y Clemente, A. (2015). Prebióticos; concepto, propiedades y efectos beneficiosos. [En línea]. [Fecha de consulta: 18 de febrero de 2016]. Disponible en: http://www.redalyc.org/articulo.oa?id=309238517015

Dahl, W. J., Foster, L. M. y Tyler, R. T. (2012). Review of the health benefits of peas (Pisum sativum L.). British Journal of Nutrition, 108, S1, S3-S10. http://doi.org/10.1017/S0007114512000852

Dahl, W. J. y Stewart, M. L. (2015). Position of the Academy of Nutrition and Dietetics: Health Implications of Dietary Fiber. Journal of the Academy of Nutrition and Dietetics, 115, 11, pp. 18611870. http://doi.org/10.1016/j.jand.2015.09.003

Duranti, M. (2006). Grain legume proteins and nutraceutical properties. Fitoterapia, 77, 2, pp. 67-82. http://doi.org/10.1016/j. fitote.2005.11.008

Espinosa-Martos, I. y Rupérez, P. (2006). Soybean oligosaccharides. Potential as new ingredients in functional food. Nutrición Hospitalaria, 21, 1, pp. 92-96.

FAO (2013). Dietary protein quality evaluation in human nutrition. FAO Food and Nutrition paper 92, FAO, Rome.

FAOSTAT. (2015). [En línea]. Disponible en: http://faostat3.fao.org/ download/Q/QC/S

Fernández, J., Redonde-Blanco, S., Villar, C., Clemente, A. y Lombó, F. (2015). Healthy effects of prebiotics and their metabolites against intestinal diseases and colorectal cancer. AIMS MICROBIOLOGY, I, pp. 48-71. http://doi.org/doi: 10.3934/microbiol.2015.1.48

Flint, H. J., Scott, K. P., Louis, P. y Duncan, S. H. (2012). The role of the gut microbiota in nutrition and health. Nature Reviews Gastroenterology y Hepatology, 9, 10, pp. 577-589. http://doi. org/10.1038/nrgastro.2012.156

Government of Canada, H. C. (2007, February 5). Eating Well with Canada's Food Guide. 
Guillon, F. y Champ, M. M.-J. (2002). Carbohydrate fractions of legumes: uses in human nutrition and potential for health. British Journal of Nutrition, 88, S3, pp. 293-306. http://doi. org/10.1079/BJN2002720

Hermsdorff, H. H. M., Zulet, M. Á., Abete, I. y Martínez, J. A. (2011). A legume-based hypocaloric diet reduces proinflammatory status and improves metabolic features in overweight/obese subjects. European Journal of Nutrition, 50, 1, pp. 61-69. http://doi. org/10.1007/s00394-010-0115-x

Hoppner, K. y Lampi, B. (1993). Folate retention in dried legumes after different methods of meal preparation. Food Research International, 26, 1, pp. 45-48. http://doi.org/10.1016/09639969(93)90104-Q

Hosseinpour-Niazi, S., Mirmiran, P., Hedayati, M. y Azizi, F. (2015). Substitution of red meat with legumes in the therapeutic lifestyle change diet based on dietary advice improves cardiometabolic risk factors in overweight type 2 diabetes patients: a cross-over randomized clinical trial. European Journal of Clinical Nutrition, 69, 5, pp. 592-597. http://doi.org/10.1038/ejcn.2014.228

Jenkins, D. J. A., Kendall, C. W. C., Augustin, L. S. A., Mitchell, S., Sahye-Pudaruth, S., Blanco Mejia, S., Chiavaroli, L., Mirrahimi, A., Ireland, C., Bashyam, B., Vidgen, E., de Souza, R. J., Sievenpiper, J. L., Coveney, J., Leiter, L. A. y Josse, R. G. (2012). Effect of Legumes as Part of a Low Glycemic Index Diet on Glycemic Control and Cardiovascular Risk Factors in Type 2 Diabetes Mellitus: A Randomized Controlled Trial. Archives of Internal Medicine, 172, 21, pp. 1653-1660. http://doi.org/10.1001/2013.jamainternmed.70

Jukanti, A. K., Gaur, P. M., Gowda, C. L. L. y Chibbar, R. N. (2012). Nutritional quality and health benefits of chickpea (Cicer arietinum L.): a review. British Journal of Nutrition, 108, S1, S11-S26. http://doi.org/10.1017/S0007114512000797

Kirpitch, A. R. y Maryniuk, M. D. (2011). The 3 R's of glycemic index: recommendations, research, and the real world. Clinical Diabetes, 29, pp. 155-159. http://doi.org/10.2337/diaclin.29.4.155

Kwon, D. Y., Daily, J. W., Kim, H. J. y Park, S. (2010). Antidiabetic effects of fermented soybean products on type 2 diabetes. $\mathrm{Nu}$ trition Research, 30, 1, pp. 1-13. http://doi.org/10.1016/j.nutres.2009.11.004

Lee, D. S. y Lee, S. H. (2001). Genistein, a soy isoflavone, is a potent alpha-glucosidase inhibitor. Febs Letters, 501, 1, pp. 84-86. http://doi.org/10.1016/S0014-5793(01)02631-X

Lovati, M. R., Manzoni, C., Castiglioni, S., Parolari, A., Magni, C. y Duranti, M. (2012). Lupin seed $\gamma$-conglutin lowers blood glucose in hyperglycaemic rats and increases glucose consumption of HepG2 cells. British Journal of Nutrition, 107, 01, pp. 67-73. http://doi.org/10.1017/S0007114511002601

Lunde, M. S. H., Hjellset, V. T., Holmboe-Ottesen, G. y Hostmark, A. T. (2011). Variations in postprandial blood glucose responses and satiety after intake of three types of bread. Journal of Nutrition and Metabolism, 2011, 437587. http://doi. org/10.1155/2011/437587

MAGRAMA. (2012). Valoracion_Nutricional_2012_tcm7-309599. pdf. [Fecha de consulta: 18 de febrero de 2016]. Disponible en: http://www.magrama.gob.es/es/alimentacion/temas/consumo-y-comercializacion-y-distribucion-alimentaria/Valoracion Nutricional_2012_tcm7-309599.pdf
MAGRAMA. (2015). Últimos datos de consumo alimentario - Últimos datos - Panel de consumo alimentario - Consumo y comercialización y distribucion alimentaria - Alimentación - .es. http:// www.magrama.gob.es/es/alimentacion/temas/consumo-y-comercializacion-y-distribucion-alimentaria/panel-de-consumoalimentario/ultimos-datos/

Marín-Manzano, M. C., Ruiz, R., Jiménez, E., Rubio, L. A. y Clemente, A. (2009). Anti-carcinogenic soyabean Bowman-Birk inhibitors survive faecal fermentation in their active form and do not affect the microbiota composition in vitro. British Journal of Nutrition, 101, 07, pp. 967-971. http://doi.org/10.1017/ S0007114508057590

Martínez-Maqueda, D., Miralles, B., Recio, I. y Hernández-Ledesma, B. (2012). Antihypertensive peptides from food proteins: a review. Food y Function, 3, 4, pp. 350-361. http://doi.org/10.1039/ c2fo10192k

Matthews, R. H. (1989). Legumes: chemistry, technology, and human nutrition. Nueva York: Marcel Dekker Inc.

McCrory, M. A., Hamaker, B. R., Lovejoy, J. C. y Eichelsdoerfer, P. E. (2010). Pulse Consumption, Satiety, and Weight Management. Advances in Nutrition: An International Review Journal, 1, 1, pp. 17-30. http://doi.org/10.3945/an.110.1006

Messina, M. J. (1999). Legumes and soybeans: overview of their nutritional profiles and health effects. American Journal of Clinical Nutrition, 70, 3, 439S-450S.

Messina, V. (2014). Nutritional and health benefits of dried beans. American Journal of Clinical Nutrition, 100, Supplement_1, 437S-442S. http://doi.org/10.3945/ajcn.113.071472

Mitchell, D. C., Lawrence, F. R., Hartman, T. J. y Curran, J. M. (2009). Consumption of Dry Beans, Peas, and Lentils Could Improve Diet Quality in the US Population. Journal of the American Dietetic Association, 109, 5, pp. 909-913. http://doi.org/10.1016/j. jada.2009.02.029

Morel, F. B., Dai, Q., Ni, J., Thomas, D., Parnet, P. y Fanca-Berthon, P. (2015). Galacto-oligosaccharides Dose-Dependently Reduce Appetite and Decrease Inflammation in Overweight Adults. Journal of Nutrition, 145, 9, pp. 2052-2059. http://doi.org/10.3945/ jn.114.204909

Nestel, P., Cehun, M. y Chronopoulos, A. (2004). Effects of long-term consumption and single meals of chickpeas on plasma glucose, insulin, and triacylglycerol concentrations. American Journal of Clinical Nutrition, 79, 3, pp. 390-395.

Neugart, S., Rohn, S. y Schreiner, M. (2015). Identification of complex, naturally occurring flavonoid glycosides in Vicia faba and Pisum sativum leaves by HPLC-DAD-ESI-MSn and the genotypic effect on their flavonoid profile. Food Research International, 76, pp. 114-121. http://doi.org/10.1016/j.foodres.2015.02.021

Nowicka, G., Klosiewicz-Latoszek, L., Sirtori, C. R., Arnoldi, A. y Naruszewicz, M. (2006). Lupin proteins in the treatment of hypercholesterolemia. Atherosclerosis Supplements, 7, 3, 477. http:// doi.org/10.1016/S1567-5688(06)81910-0

Olmedilla Alonso, B., Farré Rovir, R., Asensio Vegas, C. y Martín Pedrosa, M. (2010). Papel de las leguminosas en la alimentación actual. Actividad Dietética, 14, 2, pp. 72-76. http://doi. org/10.1016/S1138-0322(10)70014-6 
Osorio-Díaz, P., Agama-Acevedo, E., Mendoza-Vinalay, M., Tovar, J. y Bello-Pérez, L. A. (2008). Pasta added with chickpea flour: Chemical composition, in vitro starch digestibility and predicted glycemic index. Ciencia y Tecnologia Alimentaria , 6, 1, pp. 6-12.

Papanikolaou, Y. y Fulgoni, V. L. (2008). Bean Consumption Is Associated with Greater Nutrient Intake, Reduced Systolic Blood Pressure, Lower Body Weight, and a Smaller Waist Circumference in Adults: Results from the National Health and Nutrition Examination Survey 1999-2002. Journal of the American College of Nutrition, 27, 5, pp. 569-576.

Pittaway, J. K., Robertson, I. K. y Ball, M. J. (2008). Chickpeas May Influence Fatty Acid and Fiber Intake in an Ad Libitum Diet, Leading to Small Improvements in Serum Lipid Profile and Glycemic Control. Journal of the American Dietetic Association, 108, 6, pp. 1009-1013. http://doi.org/10.1016/j.jada.2008.03.009

Pusztai, A. (2008). Uses of plant lectins in bioscience and biomedicine. Frontiers in Bioscience, 13, 13, 1130. http://doi. $\operatorname{org} / 10.2741 / 2750$

Rababah, T. M., Brewer, S., Yang, W., Al-Mahasneh, M., Al-U'Datt, M., Rababa, S. y Ereifej, K. (2012). Physicochemical properties of fortified corn chips with broad bean flour, chickpea flour or isolated soy protein: fortification of corn chips. Journal of Food Quality, 35, 3, pp. 200-206. http://doi.org/10.1111/j.17454557.2012.00440.x

Roberfroid, M., Gibson, G. R., Hoyles, L., McCartney, A. L., Rastall, R., Rowland, I., Volvers, D., Watzl, B., Szajewska, H., Stahl, B., Guarner, F., Respondek, F., Whelan, K., Coxam, V., Davicco, M. J., Léotoing, L., Wittrant, Y., Delzene, N. M., Cani, P. D., Neyrink, A. M. y Meheust, A. (2010). Prebiotic effects: metabolic and health benefits. British Journal of Nutrition, 104, S2, S1-S63. http://doi. org/10.1017/S0007114510003363

Rochfort, S. y Panozzo, J. (2007). Phytochemicals for Health, the Role of Pulses. Journal of Agricultural and Food Chemistry, 55, 20, 7981-7994. http://doi.org/10.1021/jf071704w

Ros G y Periago M. J. (2005). Calidad y composición nutritiva de hortalizas, verduras y legumbres. En Gil, A. Tratado de Nutrición (Tomo II: Composición y Calidad Nutritiva de los Alimentos). Madrid: Acción Médica, pp. 229-263.

Salunke D. K. y Kadam S. S. (1989). Handbook of world food legumes: nutritional chemistry processing technology and utilization (Vol. I). Boca Ratón, Florida: CRC Press, INC.

Sandstrom, B., Hansen, L. y Sorensen, A. (1994). Pea Fiber Lowers Fasting and Postprandial Blood Triglyceride Concentrations in Humans. Journal of Nutrition, 124, 12, pp. 2386-2396.

Sarwar Gilani, G., Wu Xiao, C. y Cockell, K. A. (2012). Impact of Antinutritional Factors in Food Proteins on the Digestibility of Protein and the Bioavailability of Amino Acids and on Protein Quality. British Journal of Nutrition, 108, S2, S315-S332. http:// doi.org/10.1017/S0007114512002371

Saura-Calixto, F. y Goñi, I. (2005). Fibra dietética y antioxidantes en la dieta española y en alimentos funcionales. En: Juarez, M., Olano, A. y Moratis, F. (eds.) Alimentos funcionales. Madrid: FECYT, pp: 167-207.

Serventi, L., Chitchumroonchokchai, C., Riedl, K. M., Kerem, Z., Berhow, M. A., Vodovotz, Y., ... Failla, M. L. (2013). Saponins from Soy and Chickpea: Stability during Beadmaking and in Vitro Bio- accessibility. Journal of Agricultural and Food Chemistry, 61, 27 , pp. 6703-6710. http://doi.org/10.1021/jf401597y

Sievenpiper, J. L., Kendall, C. W. C., Esfahani, A., Wong, J. M. W., Carleton, A. J., Jiang, H. Y., Bazinet, R. P., Vidgen, E. y Jenkins, D. J. A. (2009). Effect of non-oil-seed pulses on glycaemic control: a systematic review and meta-analysis of randomised controlled experimental trials in people with and without diabetes. Diabetologia, 52, 8, pp. 1479-1495. http://doi.org/10.1007/s00125009-1395-7

Simpson, H. L. y Campbell, B. J. (2015). Review article: dietary fibremicrobiota interactions. Alimentary Pharmacology y Therapeutics, 42, 2, pp. 158-179. http://doi.org/10.1111/apt.13248

Sirtori, C. R., Triolo, M., Bosisio, R., Bondioli, A., Calabresi, L., De Vergori, V., Gomaraschi, M., Mombelli, G., Pazzucconi, F., Zacheri, C. y Arnoldi, A. (2012). Hypocholesterolaemic effects of lupin protein and pea protein/fibre combinations in moderately hypercholesterolaemic individuals. British Journal of Nutrition, 107, 8, pp. 1176-1183. http://doi.org/10.1017/S0007114511004120

Sparvoli, F., Bollini, R. y Comibelli, E. (2015). Nutritional value. En: de Ron, A. (ed.). Grain legumes. New York: Springer, pp. 291-325.

Suárez-Martínez, S. E., Ferriz-Martínez, R. A., Campos-Vega, R., Elton-Puente, J. E., de la Torre Carbot, K. y García-Gasca, T. (2016). Bean seeds: leading nutraceutical source for human health. CyTA - Journal of Food, 14, 1, pp. 131-137. http://doi.org/10.10 80/19476337.2015.1063548

Terruzzi, I., Senesi, P., Magni, C., Montesano, A., Scarafoni, A., Luzi, L. y Duranti, M. (2011). Insulin-mimetic action of conglutin- $\gamma$, a lupin seed protein, in mouse myoblasts. Nutrition, Metabolism and Cardiovascular Diseases, 21, 3, pp. 197-205. http://doi. $\operatorname{org} / 10.1016 /$ j.numecd.2009.09.004

Utrilla, M. P., Peinado, M. J., Ruiz, R., Rodríguez-Nogales, A., Algieri, F., Rodríguez-Cabezas, M. E., Clemente, A., Gálvez, J. y Rubio, L. A. (2015). Pea ( Pisum sativum L.) seed albumin extracts show anti-inflammatory effect in the DSS model of mouse colitis. Molecular Nutrition y Food Research, 59, 4, pp. 807-819. http:// doi.org/10.1002/mnfr.201400630

Varela, G. (2014). La dieta española, fortaleza y debilidades. Nutrición Clínica en Medicina, 8, pp. 109-120.

Vaz Patto, M. C., Amarowicz, R., Aryee, A. N. A., Boye, J. I., Chung, H.-J., Martín-Cabrejas, M. A. y Domoney, C. (2015). Achievements and Challenges in Improving the Nutritional Quality of Food Legumes. Critical Reviews in Plant Sciences, 34, 1-3, pp. 105-143. http://doi.org/10.1080/07352689.2014.897907

Venn, B. J. y Mann, J. I. (2004). Cereal grains, legumes and diabetes. European Journal of Clinical Nutrition, 58, 11, pp. 1443-1461. http://doi.org/10.1038/sj.ejcn.1601995

Vergara-Castañeda, H. A., Guevara-González, R. G., Ramos-Gómez, M., Reynoso-Camacho, R., Guzmán-Maldonado, H., Feregrino-Pérez, A. A., omah, B. D. y Loarca-Piña, G. (2010). Non-digestible fraction of cooked bean (Phaseolus vulgaris L.) cultivar Bayo Madero suppresses colonic aberrant crypt foci in azoxymethane-induced rats. Food $y$ Function, 1, 3, pp. 294-300. http://doi.org/10.1039/c0fo00130a

Villegas, R., Gao, Y.-T., Yang, G., Li, H.-L., Elasy, T. A., Zheng, W. y Shu, X. O. (2008). Legume and soy food intake and the incidence of type 2 diabetes in the Shanghai Women's Health Study. American Journal of Clinical Nutrition, 87, 1, pp. 162-167. 